\title{
The MicrOmega Investigation Onboard Hayabusa2
}

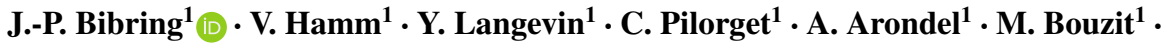 \\ M. Chaigneau ${ }^{1}$ B. Crane ${ }^{1}$ A. Darié ${ }^{1}$. C. Evesque ${ }^{1}$. J. Hansotte ${ }^{1}$ - V. Gardien ${ }^{1}$. \\ L. Gonnod ${ }^{1}$ - J.-C. Leclech ${ }^{1}$ L. Meslier ${ }^{1}$ - T. Redon ${ }^{1}$ - C. Tamiatto ${ }^{1}$ - S. Tosti ${ }^{1}$. \\ N. Thoores ${ }^{1}$
}

Received: 16 October 2015 / Accepted: 25 January 2017 / Published online: 9 March 2017

(C) The Author(s) 2017. This article is published with open access at Springerlink.com

\begin{abstract}
MicrOmega is a near-IR hyperspectral microscope designed to characterize in situ the texture and composition of the surface materials of the Hayabusa2 target asteroid. MicrOmega is implemented within the MASCOT lander (Ho et al. in Space Sci. Rev., 2016, this issue, doi:10.1007/s11214-016-0251-6). The spectral range (0.99-3.65 $\mu \mathrm{m})$ and the spectral sampling $\left(20 \mathrm{~cm}^{-1}\right)$ of MicrOmega have been chosen to allow the identification of most potential constituent minerals, ices and organics, within each $25 \mu \mathrm{m}$ pixel of the $3.2 \times 3.2 \mathrm{~mm}^{2}$ FOV. Such an unprecedented characterization will (1) enable the identification of most major and minor phases, including the potential organic phases, and ascribe their mineralogical context, as a critical set of clues to decipher the origin and evolution of this primitive body, and (2) provide the ground truth for the orbital measurements as well as a reference for the analyses later performed on returned samples.
\end{abstract}

\section{Introduction}

The Hayabusa mission, launched May 9, 2003, and samples returned to Earth by June 13, 2010, enabled the study of 25143 Itokawa, an S class asteroid (Fujiwara et al. 2006; Nakamura et al. 2011). Hayabusa2 is the JAXA follow-on mission (Yoshikawa et al. 2014), with the C-class Ryugu (formerly $1999 \mathrm{JU}_{3}$ ) asteroid the target. As an important added-on, this mission has embarked MASCOT, a small, highly integrated lander, designed to perform in situ analyses of the surface materials and of its environment (Ho et al. 2016, this issue).

MASCOT (Mobile Asteroid Surface Scout), has a limited ability to move through hopping, enabling the study of more than one site, during a limited duration (10 to 15 hours typically). With a total mass of $\sim 10 \mathrm{~kg}$ and a volume of about $0.3 \times 0.3 \times 0.2 \mathrm{~m}^{3}$, MASCOT carries four scientific instruments, for a total mass of $3 \mathrm{~kg}$ : a camera CAM (Jaumann et al. 2016, this issue), a magnetometer MAG (Hercik et al. 2016, this issue), a radiometer MARA (Grott et al. 2016, this issue), and MicrOmega, an hyperspectral microscope designed, developed and tested at IAS/Orsay, under full CNES (Centre National d'Etudes Spatiales)

\footnotetext{
$凶$ J.-P. Bibring

bibring@ias.u-psud.fr

1 Institut d'Astrophysique Spatiale (IAS), Orsay, France
} 
Table 1 Major absorption bands of minerals and organics, which could be identified by MicrOmega

\begin{tabular}{|c|c|c|c|}
\hline Wavelength range & Band width & Minerals & Assignment \\
\hline $0.99-2.30 \mu \mathrm{m}$ & $>50 \mathrm{~nm}$ & $\begin{array}{l}\text { Iron (ferrous and ferric) } \\
\text { oxides; pyroxenes; } \\
\text { olivines, Fe-bearing } \\
\text { plagioclases }\end{array}$ & $\begin{array}{l}\text { Electronic processes } \\
\text { (Crystal Feature } \\
\text { Absorption) }\end{array}$ \\
\hline $1.3-3.65 \mu \mathrm{m}$ & $10-50 \mathrm{~nm}$ & $\begin{array}{l}\text { Phyllosilicates; } \\
\text { Hydroxides; } \\
\text { Amphiboles, Hydrated } \\
\text { aluminosilica and glass; } \\
\text { AmpZeolites; } \\
\text { Carbonates; Sulfates; } \\
\text { Chlorides; Nitrates; } \\
\text { Phosphates; Perchlorates }\end{array}$ & $\begin{array}{l}\text { Combinations and } \\
\text { overtones of fundamental } \\
\mathrm{O}-\mathrm{H}, \mathrm{X}-\mathrm{OH}\left(\mathrm{X}=\mathrm{Al}^{3+}\right. \\
\left.\mathrm{Fe}^{3+}, \mathrm{Fe}^{2+}, \mathrm{Mg}^{2+}, \mathrm{Si}\right) \\
\mathrm{C}-\mathrm{O}, \mathrm{Si}-\mathrm{O}, \mathrm{N}-\mathrm{O}, \mathrm{P}-\mathrm{O}\end{array}$ \\
\hline $2.7-3.65 \mu \mathrm{m}$ & $10-50 \mathrm{~nm}$ & $\begin{array}{l}\text { Adsorbed/confined } \\
\text { water, Hydroxyl }\end{array}$ & $\begin{array}{l}\text { Fundamental and } \\
\text { overtones of } \mathrm{OH} \& \mathrm{H}_{2} \mathrm{O}\end{array}$ \\
\hline & & \multicolumn{2}{|c|}{ Organics and its assignments } \\
\hline $2.80-3.0 \mu \mathrm{m}$ & $>40 \mathrm{~nm}$ & \multicolumn{2}{|c|}{ 1st overtone of carbonyl $\mathrm{C}=\mathrm{O}$} \\
\hline $2.94-3.12 \mu \mathrm{m}$ & $>50 \mathrm{~nm}$ & \multicolumn{2}{|c|}{$\mathrm{N}-\mathrm{H}$ and $\mathrm{NH}_{2}$ group (stretch and 1 st overtone of $\mathrm{N}-\mathrm{H}$ bend) } \\
\hline$\sim 3.0 \mu \mathrm{m}$ & $>20 \mathrm{~nm}$ & \multicolumn{2}{|l|}{ Alkyne $\equiv \mathrm{C}-\mathrm{H}$ stretch } \\
\hline $3.27-3.29 \mu \mathrm{m}$ & $>20 \mathrm{~nm}$ & \multicolumn{2}{|l|}{ Aromatic $\mathrm{CH}$ stretch } \\
\hline $3.38-3.39 ; 3.41-3.42 \mu \mathrm{m}$ & $>20 \mathrm{~nm}$ & \multicolumn{2}{|c|}{ Aliphatic $\mathrm{CH}_{3} ; \mathrm{CH}_{2}$ asymmetric stretch } \\
\hline$\sim 3.45 \mu \mathrm{m}$ & $>20 \mathrm{~nm}$ & \multicolumn{2}{|l|}{$\mathrm{CH}_{2}$ Fermi resonance } \\
\hline $3.38-3.50 \mu \mathrm{m}$ & $>20 \mathrm{~nm}$ & \multicolumn{2}{|c|}{ Aliphatic $\mathrm{CH}_{3}$ asymmetric stretch } \\
\hline
\end{tabular}

support. MASCOT, as a system, has been developed by DLR in Bremen, in cooperation with CNES. MASCOT measurements will both provide unique data sets acquired in situ, and complement orbital measurements performed from the mother spacecraft, as well as serving as references for future analyses of the returned samples. MicrOmega, onboard the MASCOT lander, will have a central role in achieving these objectives. In particular, MicrOmega will acquire reflectance spectra in the near-IR of $\sim 3.2 \mathrm{~mm}$-sized surface spots with a spatial sampling of $25 \mu \mathrm{m}$, thus characterizing in situ the asteroid surface mineralogical and molecular composition at a grain scale and providing ground truth for further telescopic and orbital observations of such asteroids, and relevant meteorites. The spectral range and sampling have been chosen to enable the identification of most potential constituents, as minerals (primary and altered, oxides, salts, hydrated phases), ices and organics (Table 1), down their grain scale (Table 2). MicrOmega will not merely identify the distinct families, but also the members within each of them, offering to pinpoint the specific processes of their formation. Importantly, as for the carbon-rich phases potentially identified if present at a microscopic scale, MicrOmega will ascribe the mineralogical context in which they nucleated, through the unique capability of coupling spectroscopy to imaging.

The in situ analysis of such a pristine body has the potential to identify the processes which took place, and the ingredients that formed, in the earliest times of the solar system, from the collapse of the protosolar cloud, the dynamical evolution of the protoplanetary disk, up to the collisional accretion of protoplanet and of the asteroid belt. Of special interest will be the characterization of the volatile and carbon-rich species, known to have played a major role in the evolution of a number of solar system objects, including the Earth and other inner planets. 
Table 2 Main characteristics of the MicrOmega instrument onboard Hayabusa2/MASCOT

\begin{tabular}{ll}
\hline Dimensions & $152.5(+32$ for optical head $) \times 117.5 \times 98 \mathrm{~mm}^{3}(\mathrm{MSU})$ \\
Mass & $1.78 \mathrm{~kg}(\mathrm{MSU})+0.12 \mathrm{~kg}(\mathrm{MEU})$ \\
Spectral range & $0.99-3.65 \mu \mathrm{m}$ \\
Typical spectral sampling & $20 \mathrm{~cm}^{-1}(2 \mathrm{~nm}$ at $1.0 \mu \mathrm{m}, 25 \mathrm{~nm}$ at $3.6 \mu \mathrm{m})$ \\
FOV & $3.2 \times 3.2 \mathrm{~mm}^{2}$ \\
Spatial sampling & $25 \mu \mathrm{m}$ \\
Temperature for operations & {$\left[-40,+40^{\circ} \mathrm{C}\right]$} \\
\hline
\end{tabular}

\section{Instrument Description}

\subsection{Design Overview}

MicrOmega will characterize the texture and the composition of samples at their grain scale through the acquisition of 3D $(x, y, \lambda)$ hyperspectral microscopic image-cubes. These image-cubes will be built by sequentially illuminating the samples with a monochromatic light.

This illuminating system is made of a bright white lamp and an AOTF (Acousto-Optic Tunable Filter) based dispersive system as the monochromator (Goutzoulis and Pape 1994). For a given frequency (RF) of the acoustic signal the AOTF output light beam has a specific wavelength. The sample, illuminated by this (monochromatic) light, is imaged onto a 2D $\mathrm{HgCdTe}$ detector array, cooled by a dedicated cryocooler. By sequentially scanning the RF frequency, the sample is sequentially illuminated over the spectral domain, chosen to cover $0.99 \mu \mathrm{m}$ up to $3.65 \mu \mathrm{m}$. The entire resulting 3D image-cube is built in about $25 \mathrm{~min}$ for a baseline case of 320 spectral channels, $\sim 20 \mathrm{~cm}^{-1}$ sampling, including a start-up (initialization and cooling down of the detector) of $\sim 8 \mathrm{~min}$.

MASCOT does not have a system to collect the samples and deliver them in front of MicrOmega: per design, MicrOmega will image the samples directly in contact with its front (sapphire) window, located at the top of a small conic extension protruding one of the MASCOT plane, and constituting one of the MASCOT "feet" after it landed. The position of the samples, with respect to MicrOmega optical system, is thus accurately known (Figs. 1 and 2).

MicrOmega is divided into two main parts: (1) the MicrOmega Sensor Unit (MSU) with the illumination, detection and proximity electronics parts and (2) the MicrOmega Electronic Unit (MEU) inside the MASCOT E-box (see Sect. 2.2.3) (Fig. 1).

\subsection{Subsystems Description}

\subsubsection{Illumination Unit}

The monochromatic illumination system is based on an AOTF monochromator. The broadband light source is a commercial but qualified (at IAS) long life $(\sim 40000 \mathrm{hrs})$ ultraminiaturized filament lamp. The source illuminates the entrance of the $\mathrm{TeO}_{2}$ AOTF crystal through an aspheric condenser. Two cross polarizers are used as a light trap (for the non-diffracted white beam). The AOTF has been designed and qualified to meet the spectral range requirement, from $0.99 \mu \mathrm{m}$ to $3.65 \mu \mathrm{m}$, and the spectral width requirement $\left(\sim 20 \mathrm{~cm}^{-1}\right)$, through a two-transducers miniaturized system. When an ultrasonic wave is 


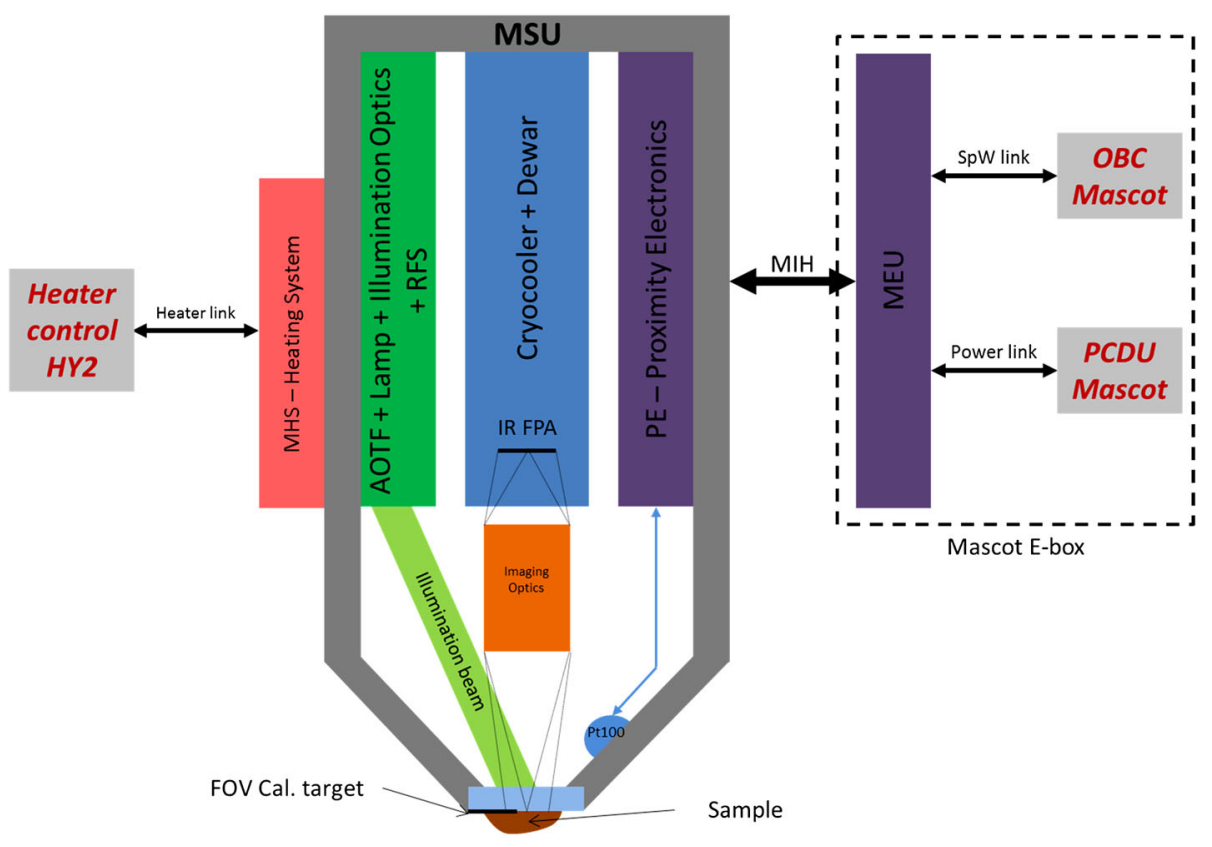

Fig. 1 Functional diagram of MicrOmega instrument onboard Hayabusa2/MASCOT

Fig. 2 MicrOmega/Hayabusa2 FM (MSU) after full integration

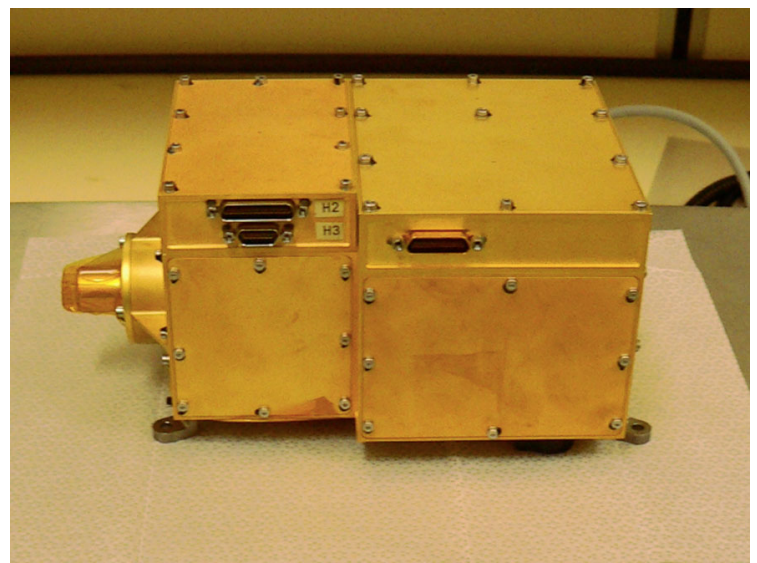

applied to the crystal, a monochromatic diffracted light exits the crystal in a fixed given direction. The wavelength of this output depends upon the frequency of the ultrasonic wave, generated by a Radio Frequency Synthesizer (RFS) in the $27 \mathrm{MHz}$ to $104 \mathrm{MHz}$ range, through piezoelectric $\mathrm{NbLiO}_{3}$ transducers (Table 3). The relationship between the wavelength of the monochromatic light generated and the ultrasonic wave frequency applied to the crystal is bijective, and has been properly calibrated, as a function of the temperature. The spectral width is constant over the $0.99-3.65 \mu \mathrm{m}$ spectral range at $20 \mathrm{~cm}^{-1}$. After exiting the AOTF, the light is directed to the MicrOmega Optical Window, in contact with the asteroid surface, through an illumination system made of two sapphire plano-convex lenses 
Table 3 Main characteristics of the MicrOmega AOTF

\begin{tabular}{ll}
\hline Material & $\mathrm{TeO}_{2}$ \\
Spectral range & $0.99 \mu \mathrm{m}-3.65 \mu \mathrm{m}$ \\
Acoustic frequency range & $27 \mathrm{MHz}-104 \mathrm{MHz}$ \\
Bandpass, FWHM & $20 \mathrm{~cm}^{-1}$ \\
Angular aperture & $\pm 3^{\circ}$ \\
Diffraction angle & $7^{\circ}$ \\
Output polarization & in the diffraction plane \\
RFS power & $\sim 2 \mathrm{~W}$ \\
\hline
\end{tabular}

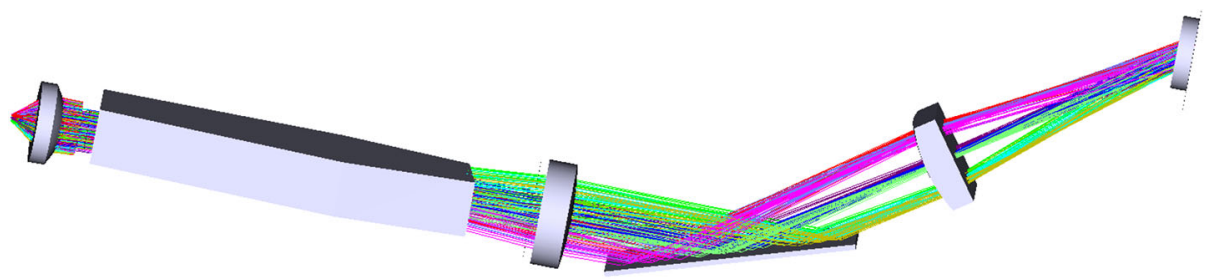

Fig. 3 Raytracing of the illumination sub-system. The light source with the aspheric condenser is on the left, whereas the MicrOmega Optical Window, in contact with the asteroid surface is on the right

and one gold coated plan mirror (Fig. 3). The output monochromatic beam is collimated on the sample, scattered with possible specific (and thus diagnostic) absorptions depending on its composition, and then imaged onto the image sensor. The scanning by steps of the RFS frequency provides the sequential illumination of the sample in all wavelengths between 0.99 to $3.65 \mu \mathrm{m}$, building a $(x, y, \lambda)$ hyperspectral cube. Since they are electrically commanded, AOTF operate without any moving part, increasing the robustness of the instrument, and give a high flexibility in the operations, with the possibility to select any spectral sequence needed, in any spectral pattern. This latter can be used to oversample spectral regions of interest in order to increase both the SNR and the spectral accuracy, for diagnostic characterization, as well as for optimizing the data budget for downlink.

\subsubsection{Imaging Unit}

The imaging optics conjugates the sample and the detector array with a magnification of 1.2 and a numerical aperture of 0.1 . The design has been optimized for the overall spectral range and the overall operating temperature range in order to increase the image quality criterion. The optical combination is a five lenses miniaturized system made of sapphire and $\mathrm{CaF}_{2}$, as shown in Figs. 4 and 5.

The sensor is the space qualified Sofradir Neptune SMW made of a $\mathrm{HgCdTe}$ detection array, hybridized on a CMOS CTIA readout circuit. The array has a size $500 \times 256 \mathrm{px}^{2}$ and a $30 \mu \mathrm{m}$ pixel pitch; for this mission, only the central area of $125 \times 128 \mathrm{px}^{2}$ is used. The HgCdTe spectral sensitivity has been tuned to cover the 0.9 to $3.7 \mu \mathrm{m}$ spectral range. It operates at $110 \mathrm{~K}$ nominally with a resulting dark current of $10 \mathrm{fA} / \mathrm{px}$. The well depth is estimated at $2.210^{6} \mathrm{e}^{-} / \mathrm{px}$. The detector is encapsulated in a hermetic Dewar, within which it is mounted on the cold part of a dedicated Ricor K508S cryocooler (with a temperature closed loop control system). Within the Dewar, a baffle, coupled to be cooled by the cryocooler, is placed in front of the detector, to strictly limit the thermal fluxes entering the detector to those coming from the samples. 


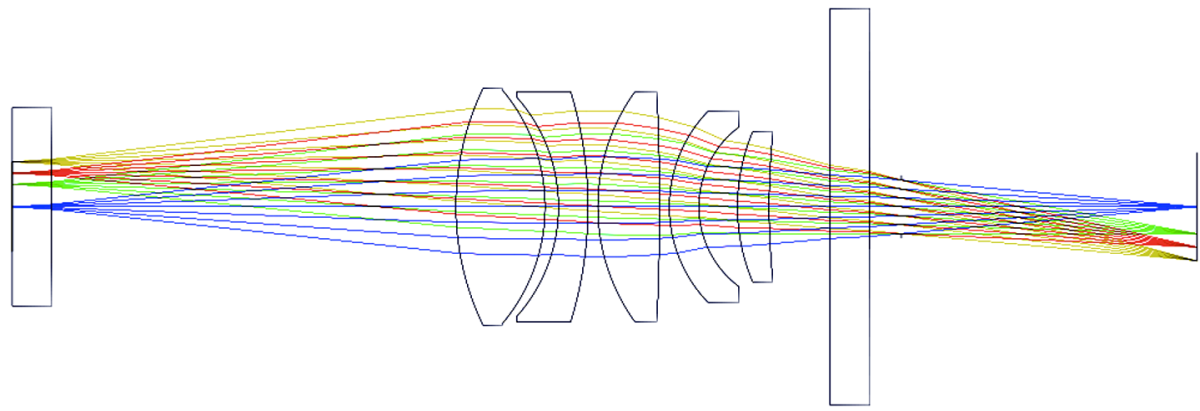

Fig. 4 Imaging optics raytracing. The MicrOmega Optical Window (sapphire), in contact with the asteroid surface, is shown on the left. The window of the detector dewar (in sapphire) is shown on the right

Fig. 5 Imaging optics assembly (height $=18.3 \mathrm{~mm}$, diameter $=$ $16 \mathrm{~mm}$ )

\subsubsection{Electronics}

Hardware Electronic functions are implemented on two boards with separated functions; one board is implemented inside the MASCOT common E-box (MicrOmega Electronic Unit /MEU) while the second (MicrOmega Proximity Electronic) is implemented inside the MSU. The MicrOmega Electronic Unit provides simple power interface between MASCOT PCDU and the instrument, the SpaceWire/data interface (nominal and redundant link) and the control of all electrical subsystems of MSU. It also manages some simple data processing and a stand-alone test mode. The MicrOmega Proximity Electronic is mounted inside the MSU, close to the analog parts of the instrument (detector, temperature sensors), to minimize the potential perturbations. There are 3 interconnect harnesses (MIH), under DLR responsibility, between MASCOT E-box and MSU. Most of lines inside these harnesses are digital lines or power lines.

The Radio Frequency Synthesizer (RFS) is also implemented within the MSU. It generates a radio frequency signal in the $27-104 \mathrm{MHz}$ range, which controls the AOTF transducers, as described above. The $20 \mathrm{kHz}$ frequency step enables to scan the light wavelength within the $0.99-3.65 \mu \mathrm{m}\left(10101-2740 \mathrm{~cm}^{-1}\right)$ range with $2 \mathrm{~cm}^{-1}$ steps. The typical RF output power is $2.0 \mathrm{~W} \pm 0.5 \mathrm{~W}$ for a power consumption $<5 \mathrm{~W}$. The use of two transducers for the AOTF enables to keep a good impedance match over the whole RF frequency range; the VSWR (voltage standing wave ratio), which characterizes the power transmission to the AOTF, remains below 1.15. 
Software To insure optimization at system level, the MicrOmega instrument has a very limited data handling and operational autonomy and relies on the MASCOT On Board Computer (MOBC) to sequence its operations and to process the data before transmission to Earth. The 3D hyperspectral data $(x, y, \lambda)$ are obtained by sequentially acquiring $2 \mathrm{D}$ images $(x, y)$ at different wavelengths $(\lambda)$. The basic interface level is the request by the MOBC of one image from MicrOmega and the transmission of the acquired data. After reception of the image, the next one is requested. The number of images and the specification of all the parameters associated with each image (e.g. integration time, summing, RF frequency) are set by the MOBC, based on a ground command defining the sequence of observations.

The 3D data cubes $(x, y, \lambda)$ are compressed onboard via the Langevin and Forni (2000) algorithm before transmission to the ground. This algorithm is based on wavelet transform and has been used successfully for OMEGA/MEx (Bibring et al. 2004) and VIRTIS/Rosetta (Coradini et al. 2007). Since image acquisition can be requested in any order, the 2D $(x, y)$ images are reorganized from the lowest to the highest wavelength before compression. The resampled 3D data cube is then split into 64 spectral channels 3D data cubes and then compressed in the $(y, \lambda)$ dimension. The data are stored in a mass memory, uplinked to the Hayabusa2 spacecraft and finally transmitted to Earth.

\subsubsection{Structure}

Mechanical Design The structure of MicrOmega Sensor Unit is machined in one block of Aluminum Alloy 6061. It is divided into three main parts: (1) the illumination part with the source and the AOTF-based monochromator, (2) the detection part with the imaging optics and the detector and its cryocooler, and (3) the electronic part.

Thermal Design The operational thermal range is $\left[-40{ }^{\circ} \mathrm{C} ;+40{ }^{\circ} \mathrm{C}\right]$ and the nonoperational thermal range $(\mathrm{NOP})$ is $\left[-50{ }^{\circ} \mathrm{C} ;+50{ }^{\circ} \mathrm{C}\right]$. As MicrOmega is an infrared sensitive instrument, the quality of science data is very sensitive to the temperature, both of the instrument and of the sample analyzed, the coldest the best. This should drive the selection of the landing site, and the sequence of science operations after landing, with possibly MicrOmega acquiring images at night.

Given the specific thermal constraints of MicrOmega, during storage and in operations, not necessarily complying with those of the other subsystems, it was chosen to decouple thermally MicrOmega from MASCOT as much as possible (Fig. 6). The thermal design is thus based on an optimized insulation of MicrOmega, and a thermal control based on survival resistors automatedly powered to maintain its temperature over a threshold of $-50{ }^{\circ} \mathrm{C}$, by the mother spacecraft. The insulation is achieved at the MicrOmega mechanical interfaces with 4 feet made of titanium. To insure the radiative decoupling, MicrOmega is covered with MLI (10 layers). Internal temperatures are monitored by 5 Pt100 sensors and the image sensor is monitored by one $2 \mathrm{n} 2222$ sensor.

\subsubsection{Flight Calibration Target}

An extended calibration plan was executed on ground, prior to integrate MicrOmega within MASCOT. Determination of transfer functions, of the variation of responses with temperature and imaging of a variety of analogues have been performed. A few examples of the later are given in Sect. 4.2 below. A summary document is under preparation, to insure an optimized reduction of the data sets acquired after landing. 


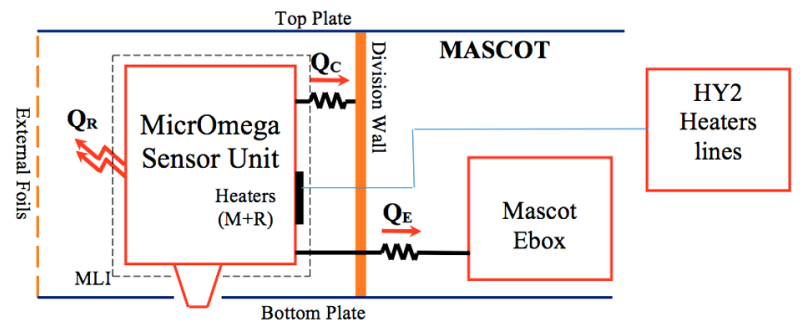

Fig. 6 Thermal concept of the MicrOmega instrument within the MASCOT lander. $Q_{\mathrm{C}}$ is the conductive power exchanged between MicrOmega and MASCOT through the mechanical interface, $Q_{\mathrm{R}}$ the radiative power exchanged between MicrOmega and MASCOT through the MLI, $Q_{\mathrm{E}}$ the conductive power exchanged between MicrOmega and the E-box though the MIH (harnesses)

However, to correct potential variations in the instrument response, due to either or both the launch campaign and the cruise phase, a calibration target has been implemented within MicrOmega, as follows: a small part of the sapphire window, representing $\sim 10 \%$ of the FOV, has been de-polished and coated with a thin layer of chromium on its external side. As a consequence, in each spectral image of a given sample, a fraction of the FOV is acquired by reflection of this calibration target, for which the spectral pattern is fully characterized, and may thus serve as a reference.

\section{Operations}

Several sets of commissioning, during cruise, were anticipated. Typically, MicrOmega should be turned $\mathrm{ON}$ once a year after insertion in the Hayabusa2 interplanetary orbit, and just after MASCOT ejection, before landing.

Upon landing, MASCOT will first get to an upright position, so that MicrOmega will face the surface of the asteroid, and perform its scientific measurements. After completion of the first science cycle, the lander will relocate and start the 2 nd science cycle. A third cycle might also be scheduled, depending on the energy available. It is planned to have MicrOmega operating at least once at each landing site, at full spatial and spectral resolution. For thermal reasons (see above), it is preferred to have MicrOmega operating during, or close to, the local nights.

\section{Performances}

MicrOmega Flight Model (FM) has been fully assembled, tested and calibrated at IAS. In particular on-ground calibration occurred in February 2014. Its performances have been fully characterized and different samples of interest have been analyzed with the instrument.

\subsection{SNR}

The Signal to Noise Ratio (SNR) and flat field have been characterized over the spectral range of the instrument $(0.99-3.65 \mu \mathrm{m})$ for different temperatures and under vacuum. Tests were performed with a Labsphere Spectralon $99 \%$ reference target. Its reflectance factor is $>99 \%$ between 1.0 and $2.0 \mu \mathrm{m}$ and $>95 \%$ between 2.0 and $2.5 \mu \mathrm{m}$ due to small absorptions. 
Fig. 7 Average SNR measured at the pixel scale over the instrument spectral range with a Labsphere Spectralon 99\% reference target, in vacuum: at $-40{ }^{\circ} \mathrm{C}$ (black); at $+25^{\circ} \mathrm{C}$ (blue). SNR estimations at each wavelength were obtained using 5 consecutive acquisitions of images with identical parameters

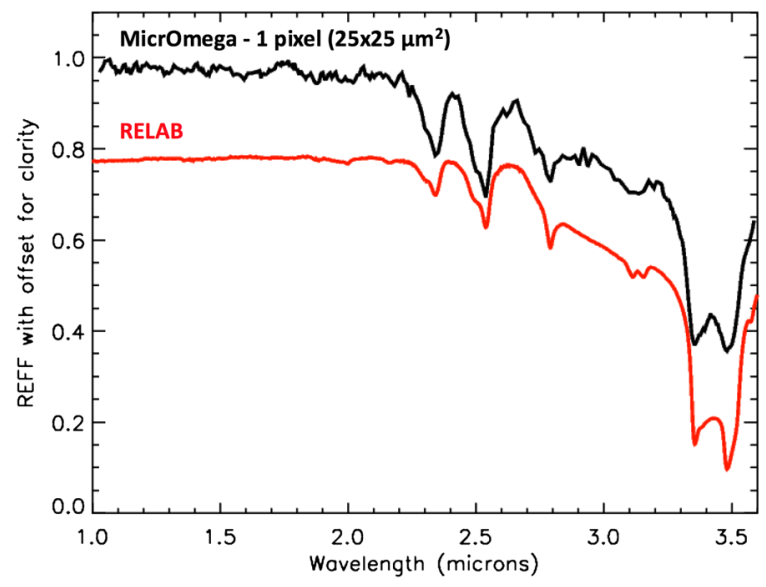

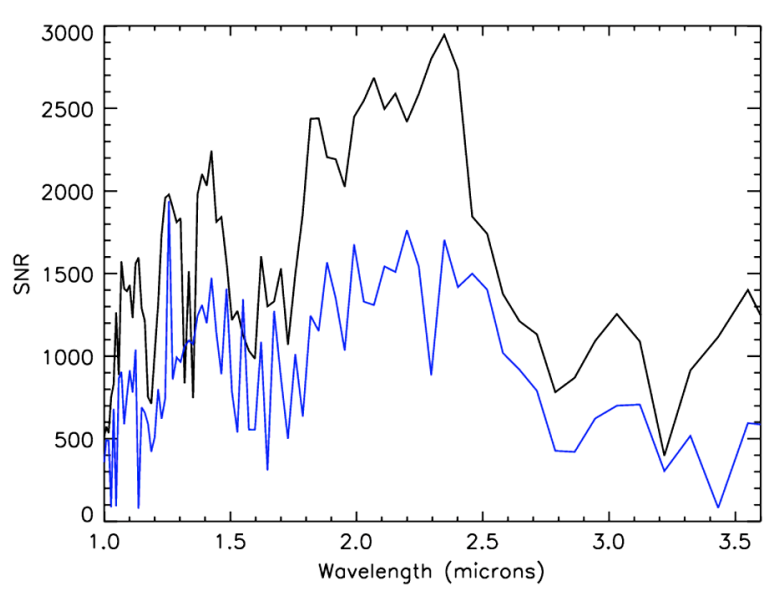

Fig. 8 Ca-carbonate powder sample (grain size: $20-50 \mu \mathrm{m}$ ) analyzed with MicrOmega FM under ambient conditions (pressure $\sim 1$ atm and instrument temperature $\sim 30{ }^{\circ} \mathrm{C}$ ). Black: reflectance spectrum obtained at the pixel scale $\left(25 \times 25 \mu \mathrm{m}^{2}\right)$ with MicrOmega FM. Red: reflectance spectrum obtained with a sample of similar composition and grain size from the RELAB/Brown University spectral library (http://www. planetary.brown.edu/relabdocs) a R at $1.3 \mu \mathrm{m}$

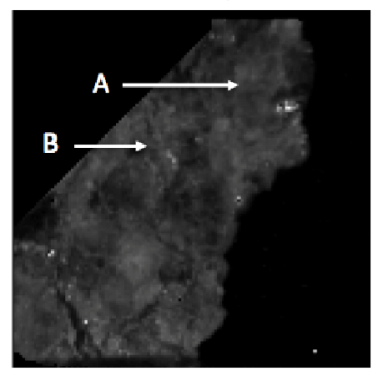

b

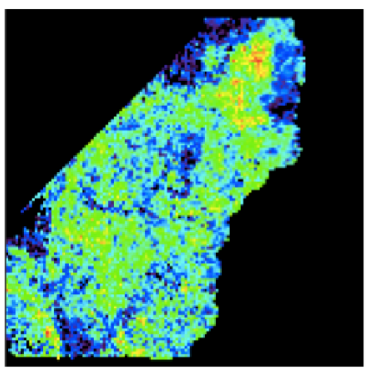

C olivine

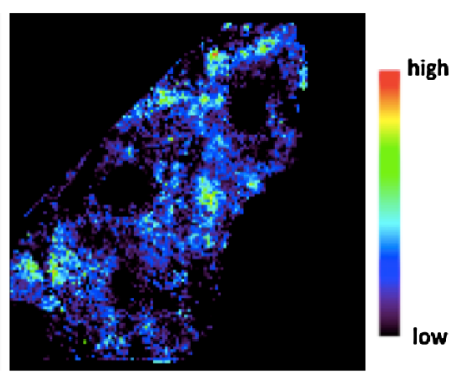

Fig. 9 Shergotty sample (small chip) analyzed with MicrOmega FM under ambient conditions (pressure $\sim 1 \mathrm{~atm}$ and instrument temperature $\sim 30^{\circ} \mathrm{C}$ ). (a) reflectance factor $(\mathrm{R})$ at a wavelength of $1.3 \mu \mathrm{m}$; (b) pyroxene map; (c) olivine map. Detection criteria are from Ody et al. (2012). Olivine always appears as a minor component mixed with medium rich Ca pyroxene (see Fig. 10) 


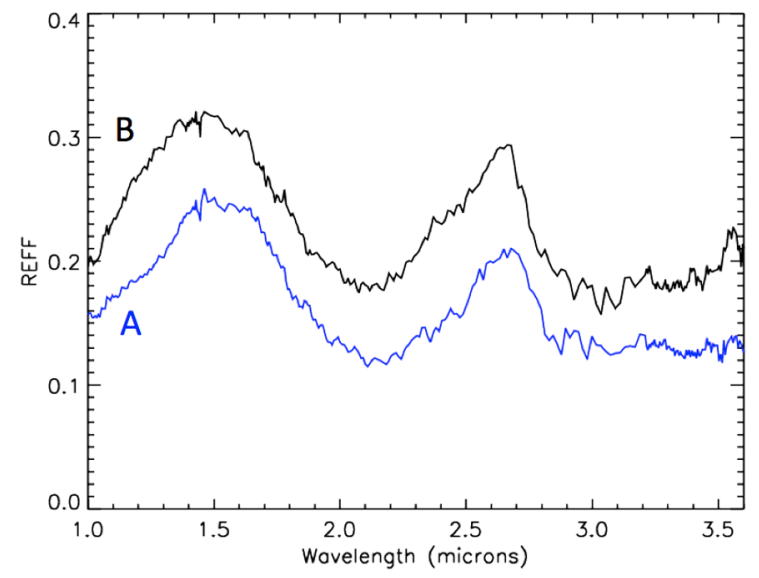

Fig. 10 Examples of spectra obtained with MicrOmega FM on the Shergotty sample (pressure $\sim 1$ atm and instrument temperature $\left.\sim 30{ }^{\circ} \mathrm{C}\right)$. Spectra were obtained at the pixel scale $\left(25 \times 25 \mu \mathrm{m}^{2}\right)$ where indicated by white arrows in Fig. 9. Spectrum A exhibits a strong signature of pyroxene mixed with olivine (presence of olivine shown by the inflection at $\sim 1.25 \mu \mathrm{m}$ and shift of the local maximum at $\sim 1.55 \mu \mathrm{m}$ ), whereas spectrum B exhibits a strong pyroxene signature (medium rich Ca pyroxene, as shown by the bands at $\sim 1.0 \mu \mathrm{m}$ and $\sim 2.1 \mu \mathrm{m})$

Stronger absorption bands are present above $2.5 \mu \mathrm{m}$, with a reflectance varying between 75 and $95 \%$. Figure 7 shows that at $-40{ }^{\circ} \mathrm{C}$, the SNR is generally above 1000 with peaks up to $\sim 3000$, considering the $16 \times$ summing mode which is used as a baseline with MicrOmega. In particular, such high SNR are measured around 3.4-3.5 $\mu \mathrm{m}$ where organics signatures are expected. SNR tends to decrease with the temperature but remains mostly above 500 with peaks up to $\sim 1500$ at $+25^{\circ} \mathrm{C}$.

Reflectance spectra are obtained after background subtraction and division by a reference signal. Although, at a microscopic scale, grains may have mean reflectance close to that of the Labsphere Spectalon 99\% used for calibration, we expect much lower reflectance for typical asteroid samples. The SNR would thus be scaled accordingly.

\subsection{Examples of Sample Characterization}

Different samples of interest have been tested with MicrOmega FM during on-ground calibration. These tests have been performed under ambient pressure $(\sim 1 \mathrm{~atm})$ and temperature (instrument temperature $\sim 30{ }^{\circ} \mathrm{C}$ ) to ease operations. Although performed under a warm environment, the results obtained during these tests are extremely satisfying, thanks to the efforts made on the design, specifically to limit the impact of environmental conditions (thermal baffle, see above). Spectral signatures obtained down to the pixel scale $\left(25 \times 25 \mu \mathrm{m}^{2}\right)$ are of high quality and clearly enable to distinguish small features down to a few percent in absorption (e.g. Fig. 8). Results from tests performed with a Ca-carbonate powder sample and a chip of Shergotty meteorite are presented in Figs. 8, 9 and 10. Figure 11 illustrates the MicrOmega capability to identify phyllosilicates and to distinguish, among them, distinct members (nontronite, montmorillonite and kaolinite), differing by their specific composition and structure, which translates distinct formation processes. 


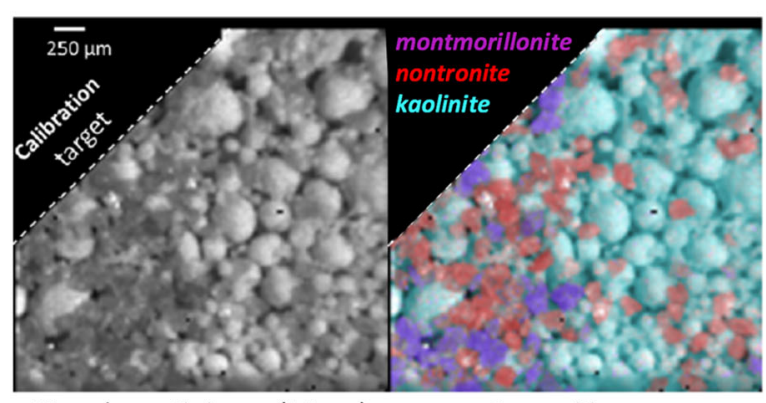

Monochromatic image $(1.3 \mu \mathrm{m})$

Composition map

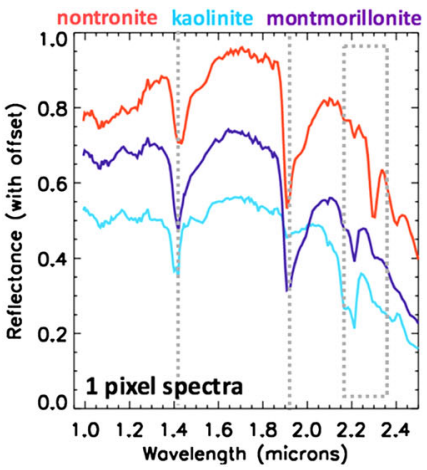

Fig. 11 Left: monochromatic image (at $1.3 \mu \mathrm{m}$ ) obtained with the MicrOmega FM of a mixture of three phyllosilicates (montmorillonite, nontronite and kaolinite). The three phyllosilicates cannot be distinguished; right: spectra acquired on grains with distinct composition (red: nontronite; blue: kaolinite; purple: montmorillonite); middle: processed image, exhibiting in false colors the three phyllosilicates, as identified through their spectra

\section{Conclusion}

MicrOmega is a near-IR hyperspectral microscope designed to characterize in situ the texture and composition of the surface materials of the Hayabusa2 target C-class asteroid Ryugu, formerly $1999 \mathrm{JU}_{3}$. Its spectral range $(0.99-3.65 \mu \mathrm{m})$ and spectral sampling $\left(20 \mathrm{~cm}^{-1}\right)$ will enable the identification of most constituent minerals, ices and organics, within each $25 \mu \mathrm{m}$ pixel of the $3.2 \times 3.2 \mathrm{~mm}^{2}$ FOV at each site in which MASCOT will come to rest. These analyzes should give critical clues to characterize the formation and evolution processes of this primitive body. MicrOmega/Hayabusa2 constitutes, through its high performances, small size $\left(\mathrm{a}\right.$ few $\left.\mathrm{dm}^{3}\right)$ and mass $(<2 \mathrm{~kg})$ a new step into the development of highly miniaturized in situ instruments for the characterization of planetary objects.

As compared to the pioneering version of MicrOmega developed for the Phobos Grunt Russian mission, Micromega/Hayabusa 2 is much more compact, by almost a factor of 2 , in both mass and volume. This Hayabusa2 instrument should be followed by a still improved version of MicrOmega to be flown on the ESA ExoMars rover (http://exploration.esa.int/ mars/45082-rover-scientific-objectives/), which will enable, within similar mass, volume and power resources, to acquire spectral images of $256 \times 256$ pix $^{2}$, and adding to the NIR spectral range 4 VIS LEDs.

Acknowledgements MicrOmega has been developed under CNES support, deeply thanked here. CNES also provides the postdoctoral funding support of C. Pilorget.

Open Access This article is distributed under the terms of the Creative Commons Attribution 4.0 International License (http://creativecommons.org/licenses/by/4.0/), which permits unrestricted use, distribution, and reproduction in any medium, provided you give appropriate credit to the original author(s) and the source, provide a link to the Creative Commons license, and indicate if changes were made.

\section{References}

J.-P. Bibring, A. Soufflot, M. Berthé, Y. Langevin, P. Drossart, M. Bouyé, M. Combes, P. Puget, A. Semery, G. Bellucci, V. Formisano, V. Moroz, V. Kottsov, OMEGA: observatoire pour la Minéralogie, l'Eau, les Glaces et l'Activité, in Mars Express: The Scientific Payload. ESA SP, vol. 1240 (2004), pp. 37-49 
A. Coradini et al., An imaging spectrometer for the Rosetta mission. Space Sci. Rev. 128, 529-559 (2007). doi:10.1007/s11214-006-9127-5

A. Fujiwara, J. Kawagushi, D. Yeomans, M. Abe, T. Mukai, J. Saito, H. Yano, M. Yoshikawa, D. Scheeres, The rubble-pile asteroid Itokawa as observed by Hayabusa. Science 312, 1330-1334 (2006)

A.P. Goutzoulis, D.R. Pape, Design and Fabrication of Acousto-Optic Devices (Dekker, New York, 1994)

M. Grott, J. Knollenber, B. Borgs, F. Hänschke, E. Kessler, J. Helbert, A. Maturilli, N. Müller, The MASCOT radiometer MARA for the HAYABUSA 2 mission. Space Sci. Rev. (2016), this special issue. doi:10.1007/s11214-016-0272-1

D. Hercik, H.-U. Auster, J. Blum, K.-H. Fornacon, M. Fujimoto, K. Gebauer, C. Guttler, O. Hillenmaier, A. Hördt, I. Richter, B. Stoll, B. Weiss, K.-H. Glaßmeier, The MASCOT Magnetometer. Space Sci. Rev. (2016), this special issue. doi:10.1007/s11214-016-0236-5

T.M. Ho, V. Baturkin, R. Findlay, C. Grimm, J.T. Grundmann, C. Hobbie, E. Ksenik, C. Lange, K. Sasaki, M. Schlotterer, M. Talapina, N. Termtanasombat, E. Wejmo, L. Witte, M. Wrasmann, G. Wübbels, J. Rößler, C. Ziach, J. Biele, C. Krause, S. Ulamec, M. Lange, O. Mierheim, R. Lichtenheldt, M. Meier, J. Reill, H.-J. Sedlmayr, P. Bousquet, A. Bellion, O. Bompis, C. Cenac-Morthe, M. Deleuze, S. Fredon, E. Jurado, E. Canalias, R. Jaumann, J.-P. Bibring, K.H. Glassmeier, M. Grott, L. Celotti, F. Cordero, J. Hendrikse, T. Okada, MASCOT - the mobile asteroid surface scout onboard the HAYABUSA2 mission. Space Sci. Rev. (2016), this special issue. doi:10.1007/s11214-016-0251-6

R. Jaumann, N. Schmitz, A. Koncz, H. Michaelis, S. Schroeder, S. Mottola, F. Trauthan, H. Hoffmann, T. Roatsch, D. Jobs, J. Kachlicki, B. Pforte, R. Terzer, M. Tschentscher, S. Weisse, U. Mueller, L. PerezPrieto, B. Broll, A. Kruselburger, T.-M. Ho, J. Biele, S. Ulamec, M. Grott, J.-P. Bibring, S. Watanabe, S. Sugita, T. Okada, M. Yoshikawa et al., The camera of the MASCOT asteroid lander on board Hayabusa-2. Space Sci. Rev. (2016), this special issue. doi:10.1007/s11214-016-0263-2

Y. Langevin, O. Forni, Image and spectral image compression for four experiments on the ROSETTA and Mars Express missions of ESA, in Society of Photo-Optical Instrumentation Engineers (SPIE). Conference Series, vol. 4115 (2000), pp. 364-373

T. Nakamura, T. Noguchi, M. Tanaka, M.E. Zolenski, M. Kimura, A. Tsuchiyama, A. Nakato, T. Ogami, H. Ishida, M. Uesugi, T. Yada, K. Shirai, A. Fujimura, R. Okazaki, S.A. Sandford, Y. Ishibashi, M. Abe, T. Okada, M. Ueno, T. Mukai, M. Yoshikawa, J. Kawagushi, Itokawa dust particles: a direct link between S-type asteroids and ordinary chondrites. Science 333, 1113-1116 (2011)

A. Ody et al., Global maps of anhydrous minerals at the surface of Mars from OMEGA/MEx. J. Geophys. Res. 117, E00J14 (2012)

M. Yoshikawa, S. Watanabe, Y. Tsuda, H. Kuninaka et al. (the Hayabusa2 Project Team), Hayabusa2: the next asteroidal mission of Japan. JSASS Aerosp. Technol. Jpn. 12(ists29), 29-33 (2014) 